\title{
Some aspects of the geomagnetic response to solar wind pressure variations: a case study at low and middle latitudes
}

\author{
U. Villante and P. Di Giuseppe \\ Dipartimento di Fisica, Università and Area di Ricerca in Astrogeofisica, L'Aquila, Italy \\ Received: 16 April 2003 - Revised: 25 February 2004 - Accepted: 1 March 2004 - Published: 14 June 2004
}

\begin{abstract}
We examined geomagnetic field observations at low and middle latitudes in the Northern Hemisphere during a 50-min interval (12 May 1999), characterized by a complex behaviour of the solar wind dynamic pressure. For the entire interval, the aspects of the geomagnetic response can be organized into four groups of events which show common characteristics for the $H$ and $D$ components, respectively. The correspondence between the magnetospheric field and the ground components reveals different aspects of the geomagnetic response in different magnetic local time (MLT) sectors. For the $H$ component, the correspondence is highly significant in the dusk and night sectors; in the dawn and prenoon sectors it shows a dramatic change across a separation line that extends approximately between (6 MLT, $35^{\circ}$ ) and $\left(13 \mathrm{MLT}, 60^{\circ}\right)$. For the $D$ component, the correspondence has significant values in the dawn and prenoon regions. We propose a new approach to the experimental data analysis which reveals that, at each station, the magnetospheric field has a close correspondence with the geomagnetic field projection along an axis (M1) that progressively rotates from north/south (night events) to east/west orientation (dawn events). When projected along M1, the geomagnetic signals can be interpreted in terms of a one-dimensional pattern that mostly reflects the field behaviour observed at geostationary orbit. Several features appear more evident in this perspective, and the global geomagnetic response to the SW pressure variations appears much clearer than in other representations. In particular, the MLT dependence of the geomagnetic response is much smaller than that one estimated by previous investigations. A clear latitudinal dependence emerges in the dusk sector. The occurrence of low frequency waves at $\sim 2.8 \mathrm{mHz}$ can be interpreted in terms of global magnetospheric modes driven by the SW pulse. This event occurred in the recovery phase after the day the SW almost disappeared (11 May 1999): in this sense our results suggest a rapid recovery of almost typical magnetospheric conditions
\end{abstract}

Correspondence to: U. Villante

(umberto.villante@aquila.infn.it) soon after a huge expansion. Overshoot amplitudes, greater than in other cases, are consistent with a significant reduction of the ring current.

Key words. Magnetospheric physics (Solar windmagnetosphere interaction; Current systems; Magnetospheric configuration and dynamics)

\section{Introduction}

An interesting aspect of the relationships occurring between the solar wind (SW) and the Earth's magnetosphere is represented by the geomagnetic response to variations of the SW parameters. In particular, the Earth's arrival of SW structures, characterized by sudden variations of the dynamic pressure $\left(\Delta \mathrm{P}_{s w}\right)$ is known to be related to impulsive variations of the geomagnetic field (SIs). SIs mainly consist of sharp variations of the north/south component $(H)$; their amplitude $(\Delta H)$ is roughly proportional to $\Delta\left(\mathrm{P}_{s w}^{1 / 2}\right)$, with a coefficient that typically ranges from 13 to $34 \mathrm{nT} /(\mathrm{nPa})^{1 / 2}$ (Siscoe et al., 1968; Su and Konradi, 1975; Nishida, 1978). Similarly, the expansion of the magnetosphere, during intervals of reduced SW pressure produces, at low latitudes, an $H$ decrease that mostly reflects the magnetospheric field waveform (Araki and Nagano, 1988). Specific attention has been addressed in the scientific literature to the local time dependence of the geomagnetic response. Statistical analysis revealed that the asymptotic response of the $H$ component shows at low latitudes $\left(15^{\circ}-30^{\circ}\right)$ a weak MLT dependence (MLT being the magnetic local time), with maximum values around local noon, minimum values around midnight, and an average value of $\sim 16.5 \mathrm{nT} /(\mathrm{nPa})^{1 / 2}$ (Russell et al., 1992, 1994a). At subauroral latitudes $\left(\sim 54^{\circ}-58^{\circ}\right)$, the asymptotic response shows a different pattern, with strongly depressed (and even negative) values in the morning, and enhanced values in the afternoon $\left(\sim 30 \mathrm{nT} /(\mathrm{nPa})^{1 / 2}\right.$; Russell and Ginskey, 1995). Previous analysis of the latitudinal dependence of the asymptotic variation ( $H$ component) showed 
Table 1. List of geomagnetic observatories with geographic and corrected geomagnetic coordinates.

\begin{tabular}{|c|c|c|c|c|}
\hline \multirow[b]{2}{*}{ Station } & \multicolumn{2}{|c|}{ Geographic } & \multicolumn{2}{|c|}{ Corr. Geomagnetic } \\
\hline & Lat. & Long. & Lat. & Long. \\
\hline $\mathrm{ABG}$ & 28.62 & 72.87 & 22.81 & 145.32 \\
\hline AQU & 42.38 & 13.32 & 36.28 & 87.51 \\
\hline BDV & 49.08 & 14.02 & 44.44 & 89.62 \\
\hline BEL & 51.84 & 20.80 & 47.55 & 96.23 \\
\hline BFE & 55.63 & 11.67 & 52.07 & 89.69 \\
\hline BMT & 40.30 & 116.20 & 34.46 & 188.68 \\
\hline BOU & 40.14 & 254.76 & 49.07 & 319.52 \\
\hline BSL & 30.40 & 270.60 & 41.43 & 340.62 \\
\hline CLF & 48.02 & 2.27 & 43.50 & 79.50 \\
\hline DLR & 29.49 & 259.08 & 38.90 & 326.22 \\
\hline ESK & 55.30 & 356.80 & 52.71 & 77.52 \\
\hline FRD & 38.21 & 282.63 & 49.22 & 357.64 \\
\hline FRN & 37.09 & 240.28 & 43.04 & 303.51 \\
\hline FUR & 48.17 & 11.28 & 43.39 & 87.05 \\
\hline HAD & 51.00 & 355.50 & 47.69 & 74.93 \\
\hline HLP & 54.61 & 18.82 & 50.67 & 95.34 \\
\hline HRB & 47.86 & 18.19 & 42.99 & 92.93 \\
\hline IRT & 52.17 & 104.45 & 47.19 & 177.20 \\
\hline KAK & 36.23 & 140.18 & 29.20 & 211.65 \\
\hline LER & 60.10 & 358.80 & 57.99 & 81.27 \\
\hline LNP & 35.00 & 121.20 & 28.72 & 193.42 \\
\hline LOV & 59.34 & 17.82 & 55.88 & 96.25 \\
\hline MMB & 43.91 & 144.19 & 37.02 & 215.31 \\
\hline NCK & 47.78 & 16.43 & 42.90 & 91.38 \\
\hline NEW & 48.26 & 242.88 & 54.96 & 303.22 \\
\hline NGK & 52.07 & 12.68 & 47.96 & 89.33 \\
\hline NUR & 60.51 & 24.66 & 56.87 & 102.46 \\
\hline OTT & 45.40 & 284.45 & 56.05 & 0.89 \\
\hline SJG & 20.00 & 293.88 & 30.00 & 10.51 \\
\hline SPT & 39.55 & 4.35 & 32.38 & 79.30 \\
\hline STJ & 47.60 & 307.32 & 53.80 & 31.19 \\
\hline SUA & 45.32 & 26.25 & 40.22 & 99.67 \\
\hline THY & 43.25 & 17.89 & 37.38 & 91.70 \\
\hline TUC & 32.25 & 249.17 & 39.88 & 314.37 \\
\hline VIC & 48.52 & 236.58 & 53.85 & 296.01 \\
\hline WNG & 53.74 & 9.07 & 50.03 & 86.87 \\
\hline
\end{tabular}

a negative gradient between $\sim 5^{\circ}-50^{\circ}$, both in diurnal and nocturnal sectors (Le et al., 1993; Russell et al., 1994a, b); more recently, however, Francia and Lepidi (2002) found a positive gradient between $\sim 36^{\circ}-65^{\circ}$, in the afternoon sector. On the other hand, since early investigations (Matsushita, 1962; Nishida and Jacobs, 1962) different waveforms of the $H$ component were detected at different stations. Nowadays, the current understanding suggests a complex scenario that relates the $H$ waveform to the combined effects of the magnetopause and ionospheric current systems; the $D$ variation ( $D$ being the east/west component) is basically related to ionospheric contributions (Araki, 1994; Tsunomura, 1998).

In the present paper we discuss several aspects of the geomagnetic field variations (observed at a number of stations between low and middle latitudes in the Northern Hemisphere, Table 1) during a 50-min interval. This period is characterized by a complex behaviour of the SW dynamic pressure (i.e. a rarefaction region, with imbedded minor amplitude variations, followed by a sharp increase). This event occurred on 12 May 1999, 15:30-16:20 UT, i.e. in the recovery phase after the day the SW almost disappeared (Farrugia et al., 2000; Fairfield et al., 2001; Le et al., 2000; Papitashvili et al., 2000; Rostoker, 2000; Terasawa et al., 2000). After a prolonged interval of extremely low values (below $1 \mathrm{~cm}^{-3}$ ), the density started to recover after 11 May at $\sim 22: 00$ UT, reaching $\sim 20 \mathrm{~cm}^{-3} 12$ on May at $\sim 18: 00 \mathrm{UT}$; in the period of interest, the number density was $\sim 7.5 \mathrm{~cm}^{-3}$, and the bulk velocity $\sim 420 \mathrm{~km} / \mathrm{s}$. We examined the relations between the SW/magnetospheric structures and ground measurements. We focused specific attention on the correspondence between the magnetospheric field $(B)$ observed at geostationary orbit in the noon quadrant and geomagnetic field measurements at different sites. Following previous investigations (Russell et al., 1992, 1994a, b; Le et al., 1993; Russell and Ginskey, 1995; Francia et al., 1999, 2001), we carefully examined the aspects of the MLT and latitudinal dependence of the asymptotic response.

Separate analysis of the $H$ and $D$ component provided interesting results. Indeed, the correspondence between $B$ and $H$ is highly significant in the dusk and night sectors. In the dawn and prenoon regions it shows a dramatic variation across an oblique separation line that extends approximately between (06:00 MLT, $\left.35^{\circ}\right)$ and (13:00 MLT, $60^{\circ}$ ). Conversely, the correspondence between $B$ and $D$ has significant values in the dawn and prenoon sectors. Obviously, these features simply reflect the different role of the current systems at different sites. They suggest, however, that the usual analysis of the $H$ component alone might provide ambiguous estimates of the amplitude and modulation of the geomagnetic response. We then propose a new approach to the experimental data analysis in which the aspects of the geomagnetic response at each station are investigated after determining the direction of maximum correlation between $B$ and the ground field. This new approach reveals that the major characteristics of the low-and middle-latitude signals can be interpreted in terms of a one-dimensional pattern that mostly reflects the field observed at geostationary orbit. 
Several features appear more evident in this perspective, and the global geomagnetic response to the SW pressure variations appear much clearer than in other representations. In particular, the MLT dependence of the geomagnetic response is much smaller than that one estimated by previous analysis, and its latitudinal dependence emerges only in the dusk and night sectors.

\section{The correspondence between interplanetary and magnetospheric observations}

The interplanetary (Wind), magnetospheric (Goes 8, MLT=UT-5.05) and geomagnetic observations (L'Aquila (AQ), Italy, corrected latitude $\sim 36.2^{\circ}$, MLT $=\mathrm{UT}+1.37$ ) are compared in Fig. 1a for the time interval 09:00-24:00 UT on 12 May. At that time Wind was located at a radial geocentric distance of $\sim 44 R_{e}$. After $\sim 12: 00 \mathrm{UT}$, the SW flow is characterized by explicit variations of the dynamic pressure which find correspondence in the magnetospheric (magnitude $B$, and $B_{z}$ component) and ground field ( $H$ component). A preliminary comparison between $\Delta \mathrm{P}_{s w}$ measurements from ACE $\left(\sim 225 R_{e}\right)$ and Wind showed a close correspondence for an average delay time of $\sim 48 \pm 3 \mathrm{~min}$. It implies a radial propagation speed of $\sim 400 \pm 25 \mathrm{~km} / \mathrm{s}$, which is consistent with the average SW velocity $(418 \pm 10 \mathrm{~km} / \mathrm{s})$. On the other hand, the best correspondence between Wind $\left(\Delta\left(\mathrm{P}_{s w}^{1 / 2}\right)\right)$ and Goes $8(\Delta B)(\rho=0.90,10: 00-14: 00$ MLT, $\rho$ being the correlation coefficient) is obtained for a delay time of $\sim 12 \pm 3 \mathrm{~min}$, i.e. somewhat longer than expected $(\sim 10 \mathrm{~min})$ for a constant, $400 \mathrm{~km} / \mathrm{s}$ transit speed (Fig. 1b). This result suggests an average speed of travelling disturbances within magnetosheath appreciably lower than in the interplanetary medium; nevertheless, the uncertain dimensions of the extended magnetosphere (Fairfield et al., 2001) do not allow any quantitative evaluation of the propagation speed. The results of Fig. 1b also show a much smaller correspondence in the morning and afternoon quadrant ( $\rho=0.52,6-10$ MLT; $\rho=0.15,14: 00-$ 18:00 MLT).

A major pressure variation was observed by Wind between $\sim$ 15:17-16:07 UT. This structure is more carefully compared with the magnetospheric field observations in Fig. 1c. The step-like variation $\left(\Delta\left(\mathrm{P}_{s w}^{1 / 2}\right) \sim 0.87(\mathrm{nPa})^{1 / 2}\right)$ is preceded by a longer term $(\sim 15 \mathrm{~min})$ rarefaction region with some smaller pressure variations. Both the declining and the ascending structures find clear correspondence in the magnetospheric field observations. These observations also confirm that the magnetospheric response in the noon quadrant is much more explicit than in the dawn and dusk sectors (Kokubun, 1983; Kuwashima and Fukunishi, 1985; Sastri et al., 2001): indeed, a sharp peak-to-peak variation of $\sim 19.2 \mathrm{nT}$ was observed by Goes 8 ( 11:00 MLT, with a global rising time $\Delta \mathrm{T}$ of $\sim 10 \mathrm{~min}$ ), while the same variation was remarkably smaller $(\sim 13.8 \mathrm{nT})$ and smoother $(\Delta \mathrm{T} \sim 16 \mathrm{~min})$ at Goes 10 position $(\sim 07: 00 \mathrm{MLT})$. When related to $\Delta\left(\mathrm{P}_{s w}^{1 / 2}\right)$, Goes 8 observations suggest a normalized magnetospheric response of $\sim 22.4 \mathrm{nT} /(\mathrm{nPa})^{1 / 2}$ in
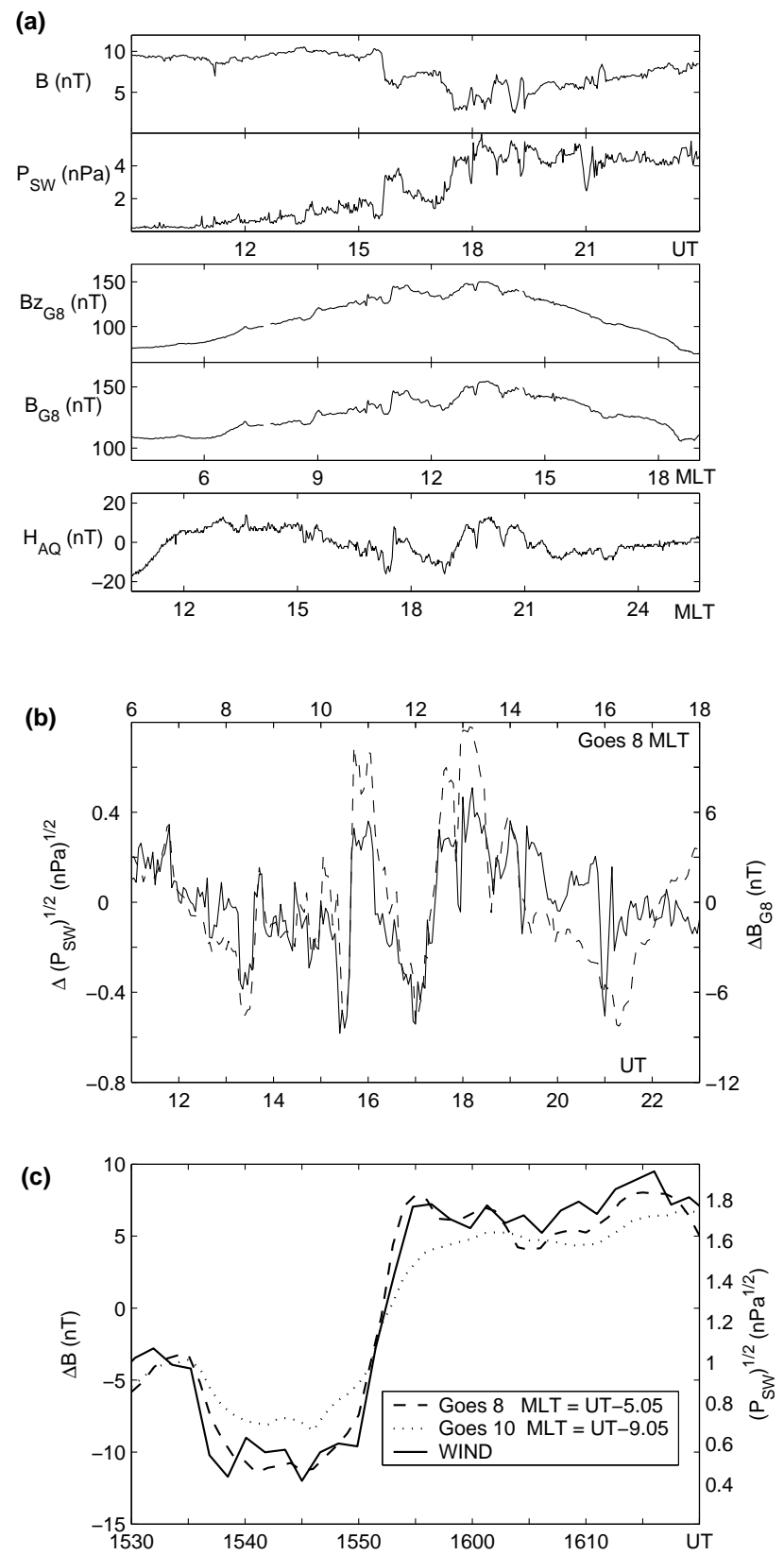

Fig. 1. (a) A comparison between Wind, Goes 8 and ground observations at L'Aquila for 12 May 1999 (09:00-24:00 UT). From the top: $B$ is the IMF intensity, $\mathrm{P}_{S W}$ the $\mathrm{SW}$ dynamic pressure, $B_{z G 8}$ the north-south component of the magnetospheric field in GSM coordinates, $\mathrm{B}_{G 8}$ the total magnetospheric field, and $\mathrm{H}_{A Q}$ the northsouth component of the geomagnetic field. Magnetospheric and ground observations are organized in Magnetic Local Time (MLT). (b) The correspondence between the 3-min averages of the square root of the SW pressure (solid line) and the magnetospheric field (dashed line) (11:00-23:00 UT). SW observations have been delayed by 12 minutes. In both cases the long-term variations have been removed. On the top scale the MLT at Goes 8. (c) A comparison between the square root of the SW pressure (solid line) and the magnetospheric field from Goes 8 (dashed line), and Goes 10 (dotted line). 

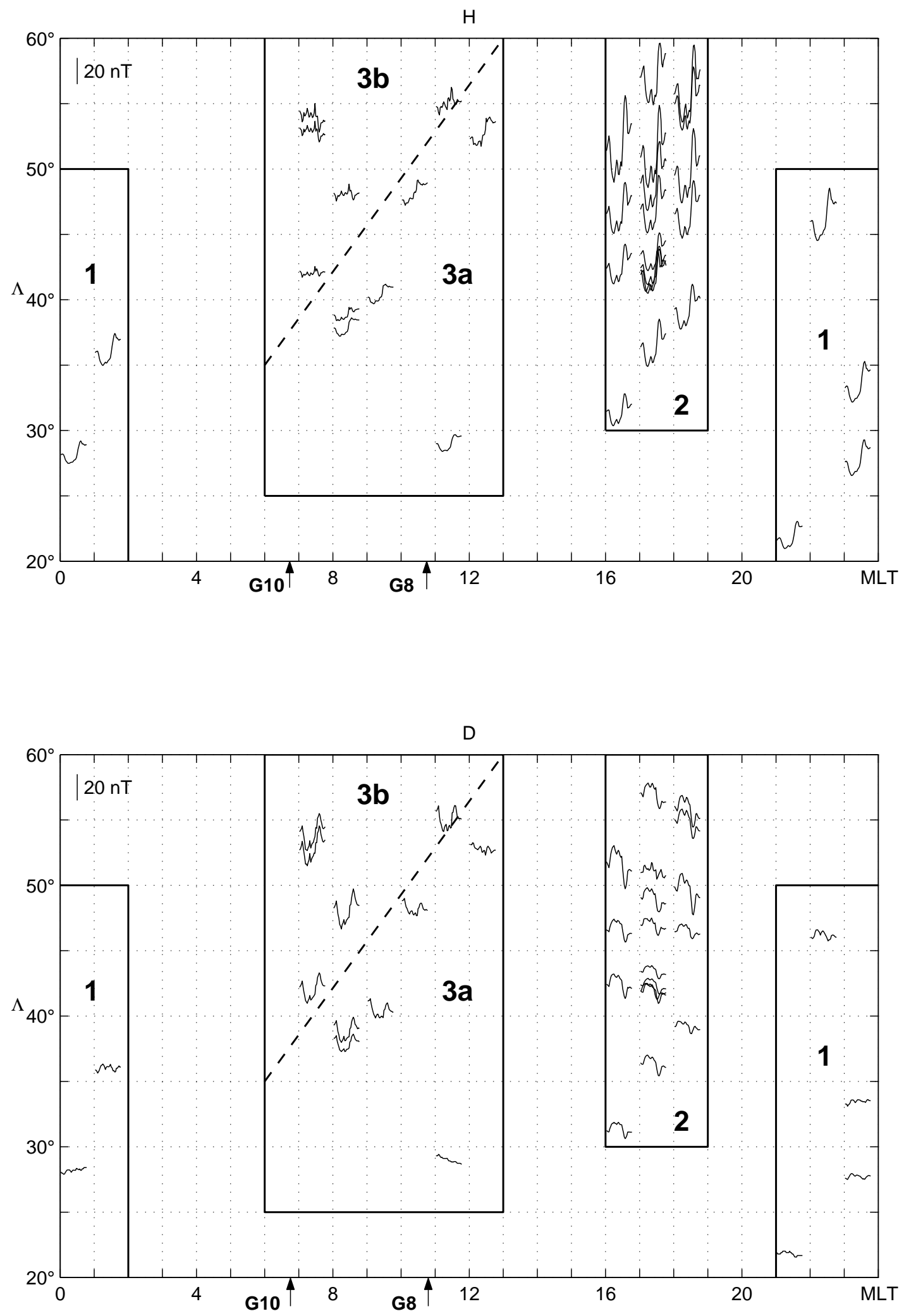

Fig. 2. Geomagnetic field observations (1-min averages) at stations located at different MLT and latitude $\Lambda$ in the Northern Hemisphere for the time interval 15:35-16:05 UT. $H$ component is in the upper panel and $D$ component in the lower panel. Ground observations are organized in four groups, 1, 2, 3a, and 3b, according to the similar behavior observed in the geomagnetic field components. The MLT of GOES satellites (G8 and G10) is marked with arrows at the bottom of both panel. 
(a)

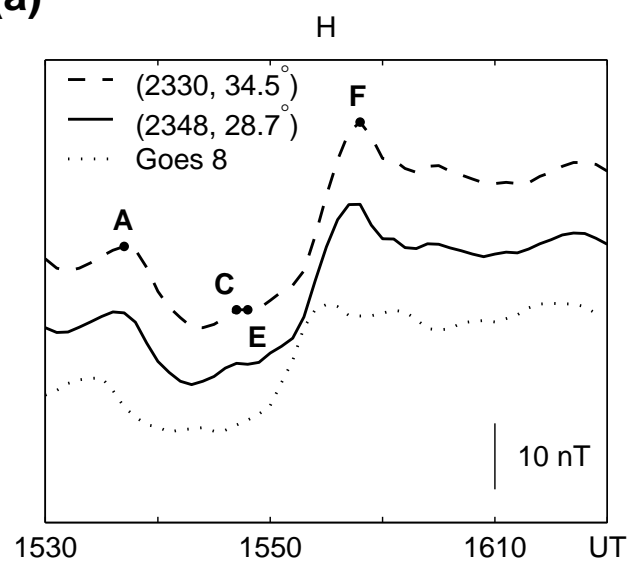

Group 1

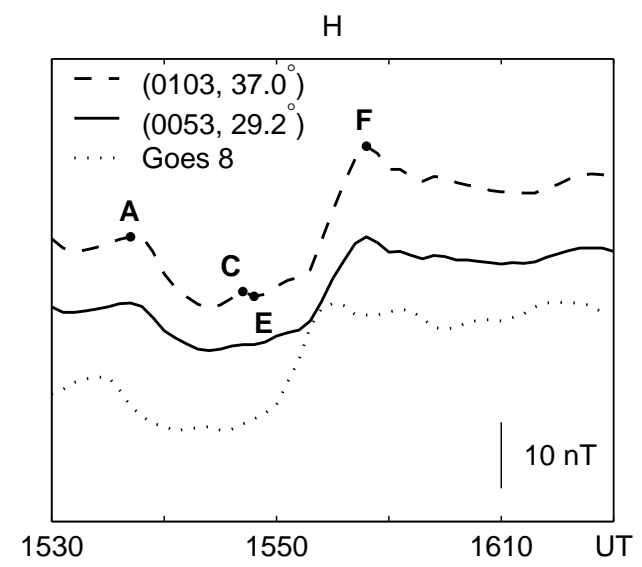

(b) $\mathrm{H}$

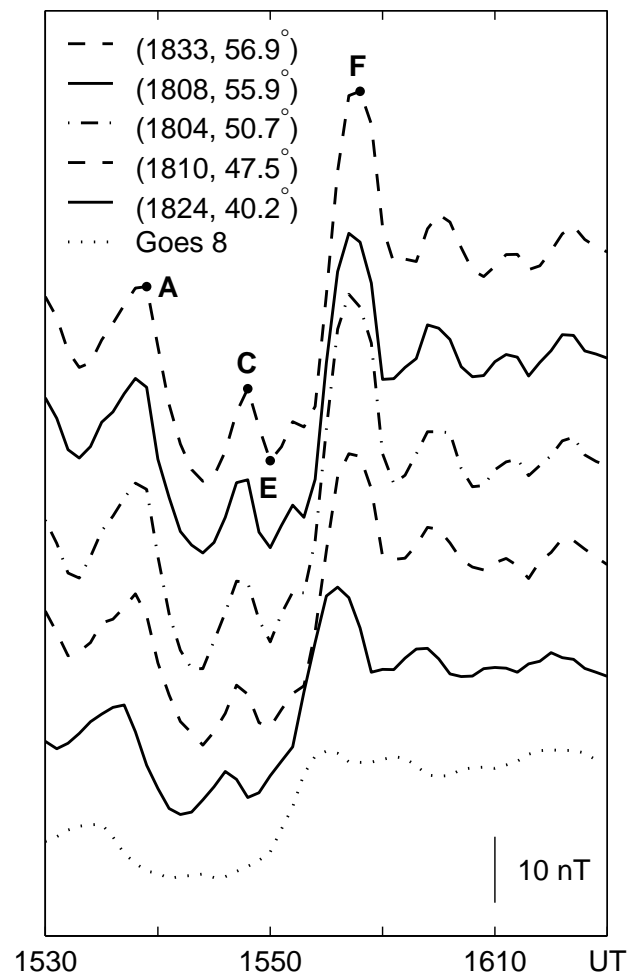

\section{Group 2}

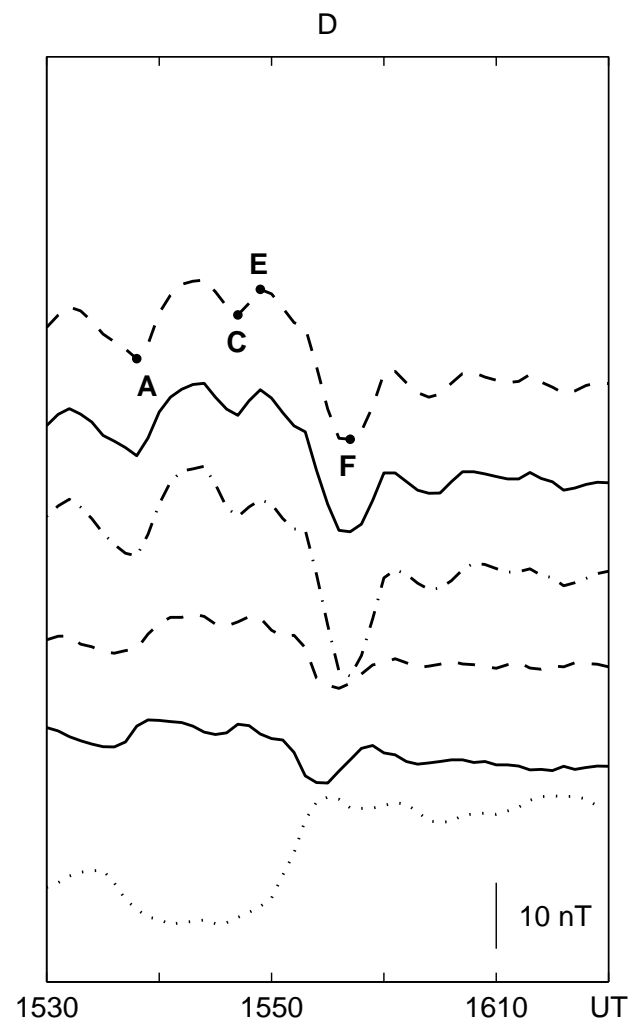

Fig. 3. (a) The magnetic field observations ( $H$ component) for two different MLT sectors in group 1. (b) The magnetic field observations for a single MLT strip in group 2. Left plot: $H$ component, right plot: $D$ component. In the bottom trace of each panel the magnetospheric field from Goes 8 .

the noon quadrant. Correspondingly, the asymptotic variation at Goes 8 and Goes 10 is, respectively, $\sim 16.0 \mathrm{nT}$ $\left(\sim 18.7 \mathrm{nT} /(\mathrm{nPa})^{1 / 2}\right)$, and $\sim 12.7 \mathrm{nT}\left(\sim 14.6 \mathrm{nT} /(\mathrm{nPa})^{1 / 2}\right)$. Similarly, the initial negative variation has a total excursion of $\sim-8.2 \mathrm{nT}$ and $\sim-4.5 \mathrm{nT}$ at Goes 8 and Goes 10. The first ground SI appearance is detected approximately within $1 \mathrm{~min}$ after the magnetospheric field variation (Nishida, 1978): this observation suggests an average propagation velocity of as- sociated disturbances in the inner magnetosphere of at least $\sim 600 \mathrm{~km} / \mathrm{s}$, i.e. consistent with current estimates of the fast mode velocity (Farrugia et al., 1989; Araki, 1994; Araki et al., 1997). As we show in the following, ground observations in any time sector mostly reflect the magnetospheric field behaviour in the noon quadrant. 
(a)

$\mathrm{H}$

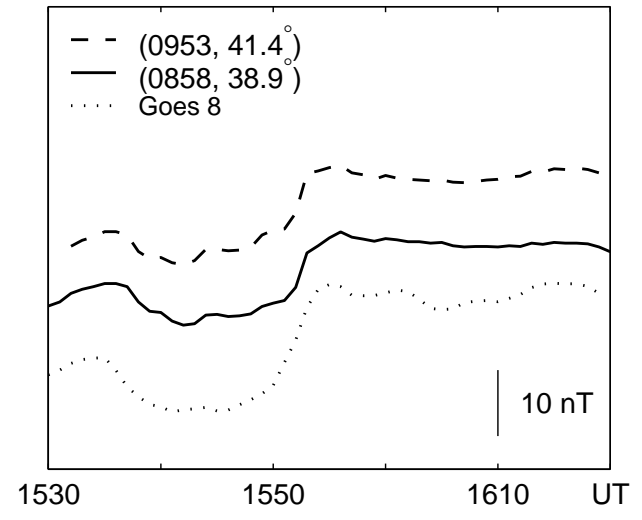

\section{Group 3a}

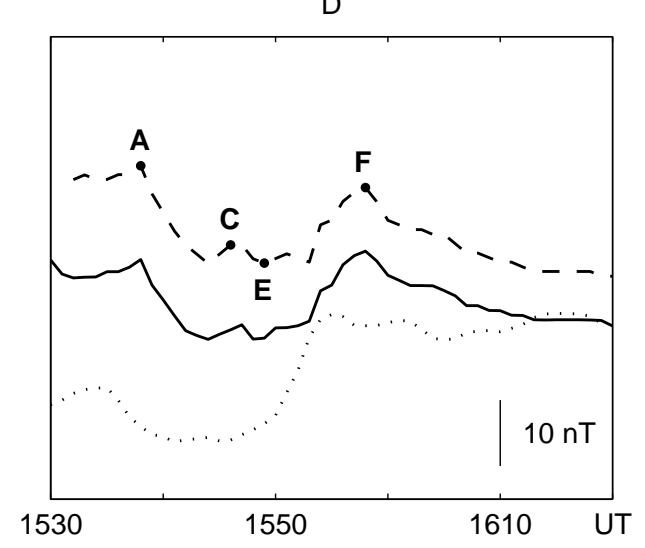

(b)

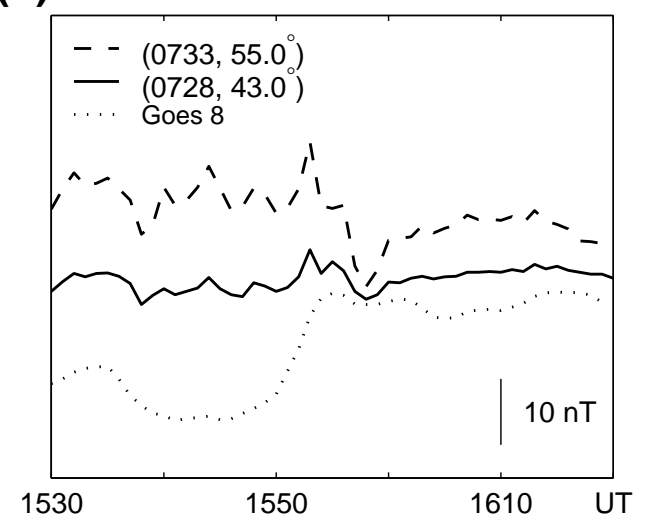

\section{Group 3b}

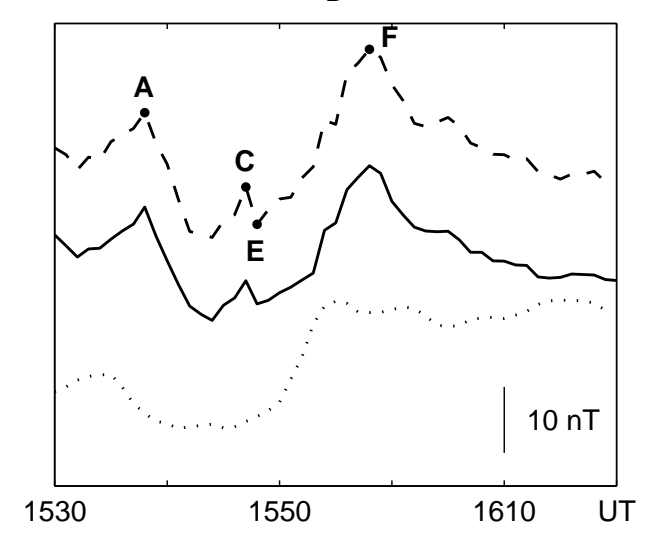

Fig. 4. The same as in Fig. $3 b$ for group $3 a(a)$ and $3 b(\mathbf{b})$.

\section{An analysis of the geomagnetic response}

A joint analysis of the morphological aspects of $H$ and $D$ for the entire 50-min interval (15:30-16:20 UT, 1-min averages, Fig. 2) shows a clear organization of ground observations in groups of events with different characteristics (Matsushita, 1962; Nishida and Jacobs, 1962). This analysis also makes explicit that a clear variation of the geomagnetic field behaviour (which mostly influences the $H$ component) occurs across a separation line that extends approximately between (06:00 MLT, $\left.35^{\circ}\right)$ and (13:00 MLT, 60 ${ }^{\circ}$ ). These different aspects are shown more clearly in Figs. 3 and 4, where observations are organized in narrow MLT strips. Here points A, C, E, and F identify the major field variations which have different amplitudes in different regions (and even disappear in some cases). Namely, A (15:36-15:39 UT) identifies the peak value before the main decrease; C (15:47-15:48 UT), the peak enhancement occurring during depressed magnetospheric field conditions; E (15:48-15:49 UT), the minimum value before the main variation; F (15:56-15:58 UT), the overshoot field (i.e. the peak value that precedes the asymptotic variation, Russell and Ginskey, 1993); we also show, for comparison, in the bottom trace of each panel, the $B$ field at Goes 8 position. The principal characteristics of the different groups can be briefly summarized as follows.

Group 1. In the midnight sector (22:00-01:00 MLT, Fig. 3a) the geomagnetic field trace is characterized by explicit variations of the $H$ component ( $D$ only shows small amplitude fluctuations, Fig. 2). These variations mostly reflect the magnetospheric field trace. No clear evidence for the $\mathrm{C}$ enhancement is detected in this sector. As for other cases, significant differences of the geomagnetic response appear for small MLT separation. Indeed, at $\sim 29^{\circ}$ the peak variation $\left(\mathrm{H}_{F}-\mathrm{H}_{E} \sim 24.2 \mathrm{nT}\right)$ at $23: 48 \mathrm{MLT}$ is $\sim 50 \%$ larger than one hour later ( $\sim 16.3 \mathrm{nT}, 00: 53 \mathrm{MLT})$.

Group 2. In the dusk sector (16:00-19:00 MLT, Fig. 3b) the traces of both components are much more structured. A clear $\mathrm{C}$ enhancement precedes by several minutes a sharp overshoot $(\mathrm{F})$. Both $\mathrm{C}$ and $\mathrm{F}$ (as well as the entire pattern) show a general tendency to increase with increasing latitude and find poor correspondence in the magnetospheric field. The most significant $H$ variations correspond to simultaneous variations of the $D$ component of opposite sign: indeed, 

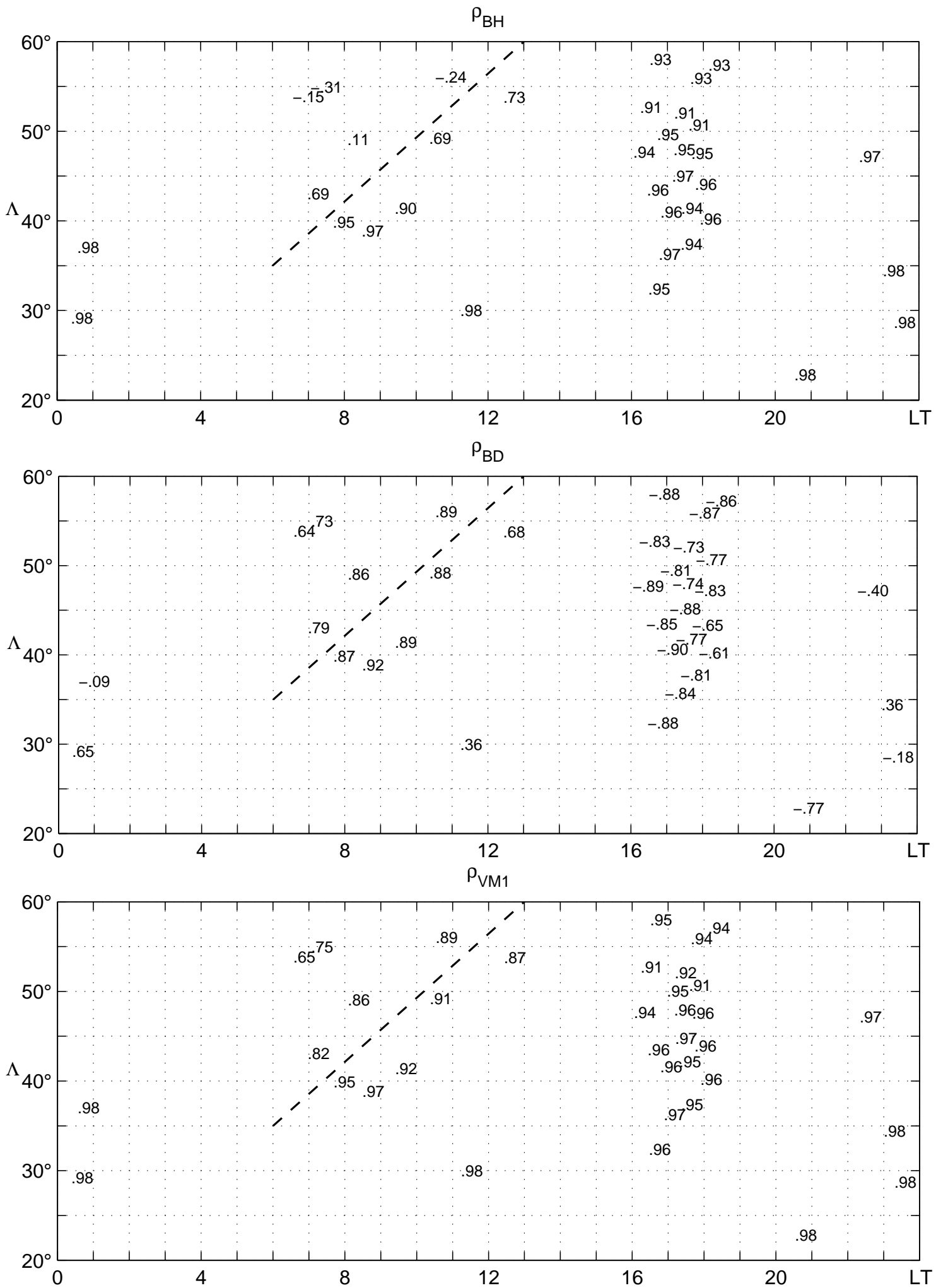

Fig. 5. The correlation coefficient between the magnetospheric field B and the geomagnetic field elements for the time interval 15:3016:20 UT. Top panel: correlation with the $H$ component $\left(\rho_{B H}\right)$; central panel: correlation with the $D$ component $\left(\rho_{B D}\right)$; bottom panel: correlation with the field projection along the maximum correlation axis $\left(\rho_{V M 1}\right)$. 
(a)

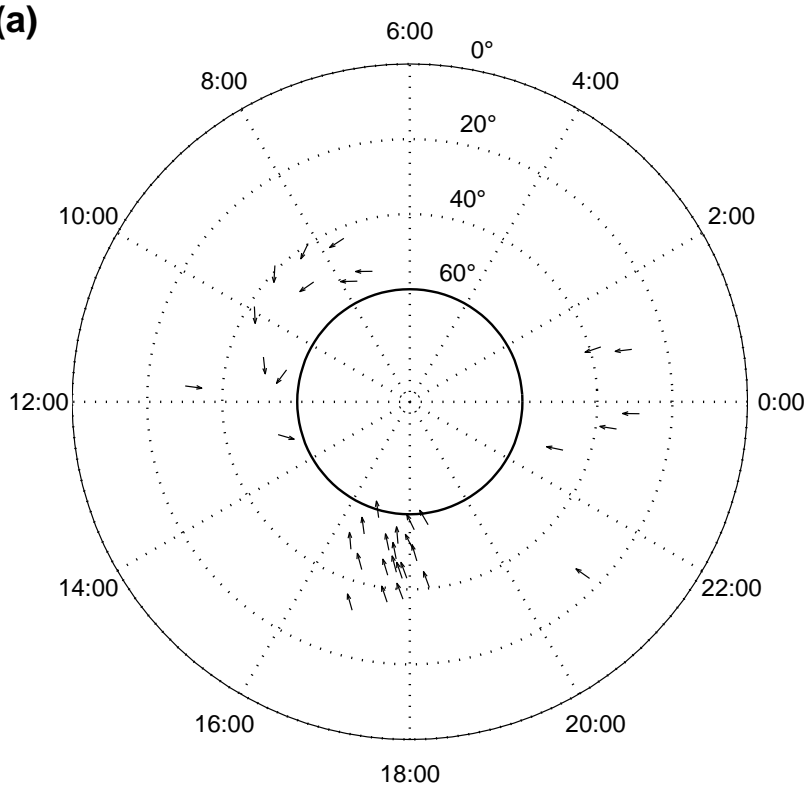

(b)

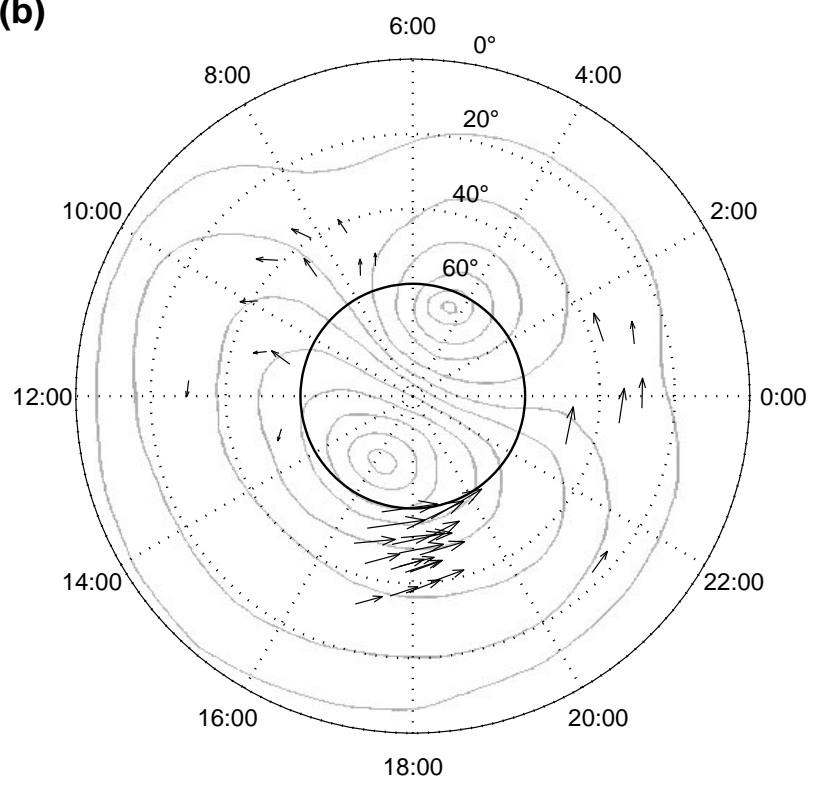

Fig. 6. (a) The orientation of the maximum correlation axis (M1) between the magnetospheric field $B$ and the geomagnetic field for the entire set of ground stations. (b) The equivalent ionospheric current system superimposed to the current system proposed by Nishida (1968). The length of the arrows is proportional to the asymptotic variation.

$D$ shows negative peak values (A), negative $\mathrm{C}$ variations, deep minimum values $(\mathrm{F})$, and a general tendency to decrease after the main variation.

Group 3. Unlike dusk and night events, dawn and prenoon events (07:00-13:00 MLT, Fig. 4) are characterized by a $D$ behaviour which basically reflects the magnetospheric field, with a similar pattern on both sides of the separation line (Fig. 2). Nevertheless, a dramatic amplitude reduction occurs at later MLTs: for example, the peak-to-peak variation is $\sim 20.9 \mathrm{nT}$ at $\left(07: 28 \mathrm{MLT}, 43^{\circ}\right)$ and $\sim 13.7 \mathrm{nT}$ at (09:53 MLT, $41.4^{\circ}$ ). Conversely, striking differences emerge in the $H$ component, even for small MLT separation. Indeed, while below the separation line (group 3a), the $H$ component still reflects the magnetospheric field, above this line (group 3b) it rather shows an irregular behaviour with a dominant positivethen-negative variation which is more explicit at $\sim 55^{\circ}$.

A simple analysis of the correlation coefficient between $B$ and the geomagnetic field components for the entire 50-min interval $\left(\rho_{B H}, \rho_{B D}\right.$, Fig. 5) reinforces the conclusions of the previous paragraph. Indeed, $\rho_{B H}$, which is high $(>.90)$ in the dusk sector, has maximum values $(>.97)$ in the midnight sector. Moreover, greater $\rho_{B H}$ values are typically observed at lower latitudes, as expected for a smaller influence of the ionospheric current system. In the dawn and prenoon sectors, significant differences occur across the separation line: indeed, below this line (i.e. at later MLTs), $\rho_{B H}$ has high values; conversely, above this line, $H$ is not correlated to $B$. The $\rho_{B D}$ values confirm a different MLT dependence: it has significant values in the dawn and prenoon sectors while showing some evidence for an anticorrelation in the dusk sector.
We adopted a different approach to the experimental data analysis to make more clear the aspects of the correspondence between the magnetospheric and ground field. For this scope, we determined the direction ( $\mathrm{V}$-axis) associated with the maximum excursion of the magnetospheric field in the noon quadrant (it is found in the meridian plane, with a strongly dominant contribution along $Z_{s m}$ ). We then identified at each station the direction (M1-axis) of maximum correlation (for the entire 50-min interval) between the magnetospheric field projection along $\mathrm{V}$ and the geomagnetic field projection along M1, evaluating the correlation coefficient $\left(\rho_{V M 1}\right)$ between the two fields at steps of $1^{\circ}$. The results of this analysis (Figure 5, bottom panel) are very interesting: indeed, they reveal a highly significant correspondence between the magnetospheric and the ground field along M1 in the entire latitudinal and MLT range. No dramatic variation of the correlation coefficient occurs in this case across the separation line. Nevertheless, the smaller $\rho_{V M 1}$, with minimum values at $\left(\sim 07: 00 \mathrm{MLT}, \sim 55^{\circ}\right)$, suggest a greater influence of ionospheric contributions at earlier MLTs.

In addition, we also determined, at steps of $1^{\circ}$, the direction (M2) of maximum asymptotic variation of the ground field. For this scope we considered, as the initial level, the 5 -min averages of the geomagnetic signals before the SI and, as the final level, the new steady state (15-min average) observed 15 min after the SI onset (different choices of the averaging intervals do not significantly influence the results of our analysis). As a matter of fact, M1 and M2 typically coincide: it confirms that at low and middle latitudes the ground variations mostly reflect, with different amplitude and orientation, the magnetospheric field variations. Nevertheless, in 


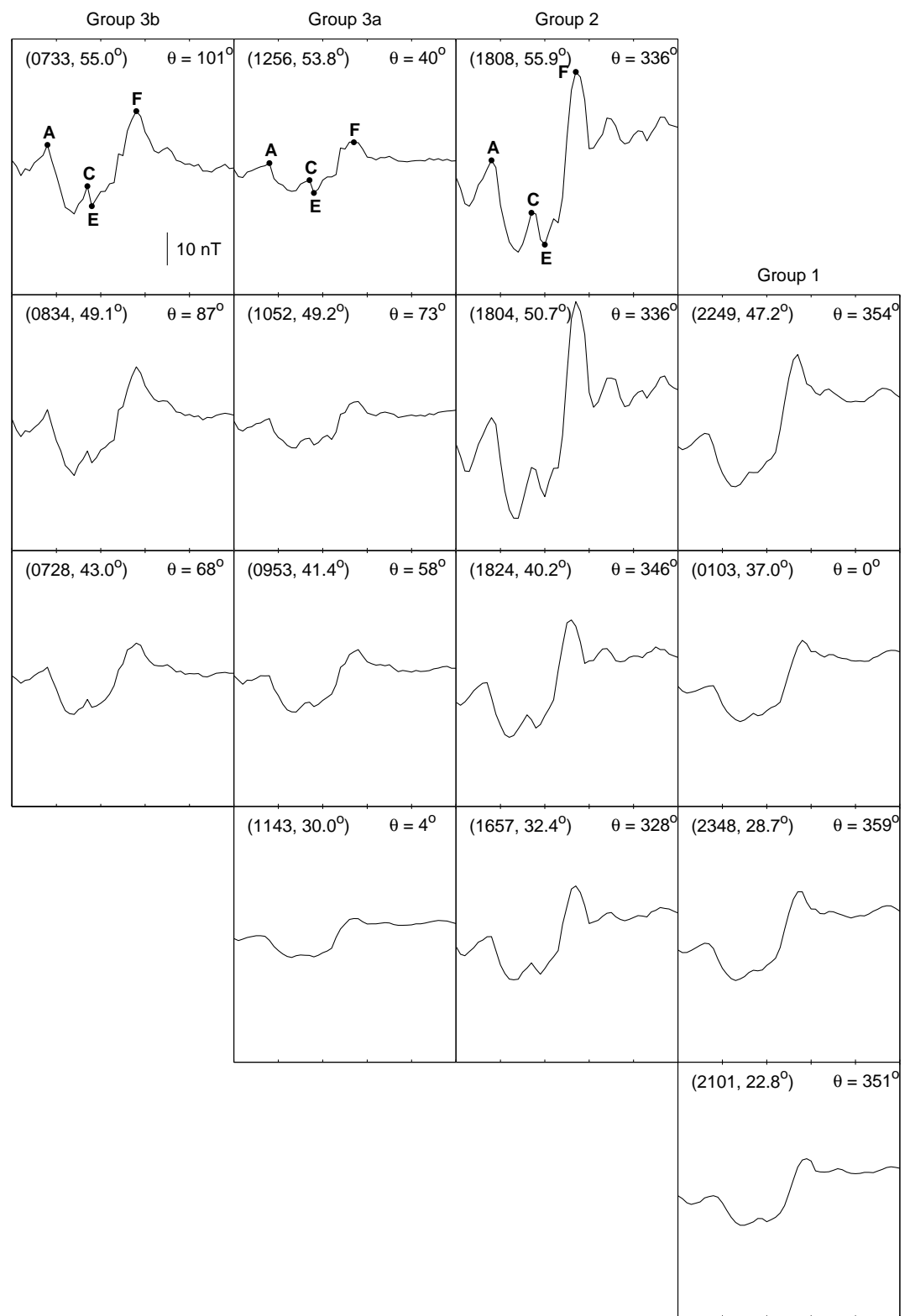

Fig. 7. A summary of the characteristics of the ground response along the M1 axis.

agreement with previous conclusions, small angular separations between $\mathrm{M} 1$ and $\mathrm{M} 2$ are observed at $\sim 7 \mathrm{MLT}, \sim 55^{\circ}$, confirming a more significant effect of ionospheric currents.

The orientation of M1 axis (Fig. 6a) shows an explicit and regular MLT dependence: it is mostly along $H$ in the night sector; it rotates westward $\left(10^{\circ}-30^{\circ}\right)$ in the dusk sector; it is progressively closer to $D$ with increasing latitude in the dawn and prenoon sectors. However, at $\left(\sim 12 \mathrm{MLT}, 30^{\circ}\right) \mathrm{M} 1$ is newly oriented along $H$. Assuming that the field perturbations are generated by a horizontal current layer in the ionosphere, the orientation of the equivalent current system can be inferred by a $90^{\circ}$ rotation of M1 (Fig. 6b). Obviously, the small latitudinal range does not allow for conclusions on the entire current system. However, the inferred currents show a general correspondence with a basic two vortex pattern as that proposed by Nishida (1968). Tentatively, we would also suggest that a closer agreement between model and observations might be obtained, translating the focus of the afternoon vortex by several degrees toward earlier MLTs; nevertheless, night observations suggest in the dark sector an extension of the vortex system to low latitudes smaller than in other cases.

Figure 7 shows, for different latitudinal strips, some examples of the geomagnetic field trace along M1. It reveals, much better than other representations, that a similar onedimensional pattern now emerges in any time sector (indeed, significant signals perpendicular to M1 occur only in some cases in the dawn sector). This pattern clearly reflects, from low to middle latitudes, the main aspects of the magnetospheric field trace. Nevertheless, at $\left(0733,55^{\circ} ; 0834,49^{\circ}\right)$ the long-term decrease after the peak value reveals a more 
(a)
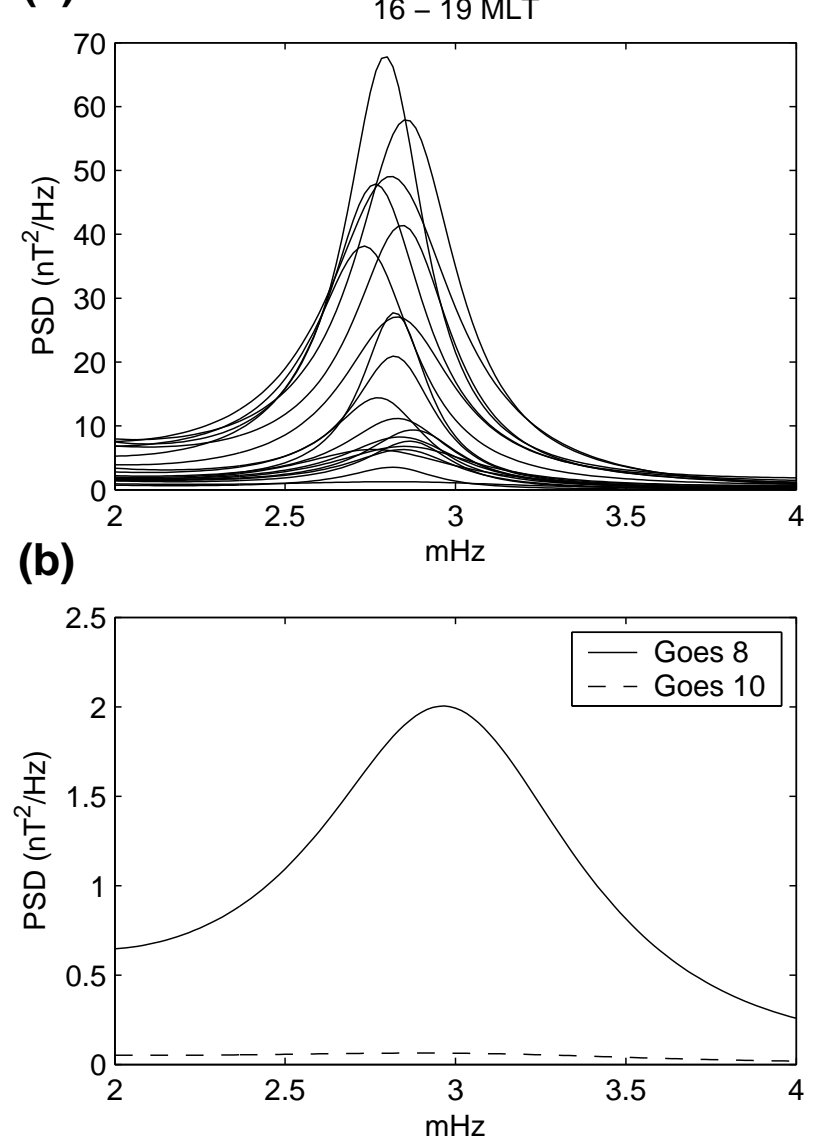

Fig. 8. (a) Spectral analysis for the time interval 16:00-16:20 UT for stations located in the dusk sector (16:00-19:00 MLT). (b) The same for Goes 8 and Goes 10 .

persistent ionospheric contribution. Independent of orientation, the global pattern, for comparable latitudes, shows maximum amplitude (and steeper variation) in the dusk sector; it progressively decreases in the midnight and dawn sectors, reaching minimum values in the prenoon quadrant. Although with different relevance, the additional A through $\mathrm{F}$ elements appear in any time sector, and are less significant below $\sim 40^{\circ}$.

Our representation may also be useful for a more definite comparison with theoretical models. For example, the effects of the Chapman-Ferraro current have been often evaluated in terms of a step-like $H$ signal decreasing with increasing latitude (Araki, 1994). Our results suggest a more complex scenario in which noon observations are characterized by an almost constant step-like response that assumes different orientations at different latitudes, while the $H$ variation detected in the dusk sector shows a positive latitudinal gradient which is in conflict with the decrease expected for the effects of the magnetopause current alone.

Dusk observations are characterized by sharp overshoots. Their amplitudes (increasing with latitude) overcome the asymptotic variations by a factor between $\sim 1.3$ and $\sim 2.0$, and are much greater $\left(\sim 70 \mathrm{nT} / \mathrm{nPa}^{1 / 2}\right.$ at $\sim 53^{\circ}$; $\sim 28 \mathrm{nT} / \mathrm{nPa}^{1 / 2}$ close to midnight at $\left.\sim 29^{\circ}\right)$ than those estimated by previous analysis $\left(\sim 18 \mathrm{nT} / \mathrm{nPa}^{1 / 2}\right.$ at low latitudes; between $\sim 17.4 \mathrm{nT}^{-\mathrm{nPa}^{1 / 2}}$ and $\sim 22.5 \mathrm{nT} / \mathrm{nPa}^{1 / 2}$ at $\sim 36^{\circ} ; 50$ $55 \mathrm{nT} / \mathrm{nPa}^{1 / 2}$ at $54^{\circ}-58^{\circ}$; Russell and Ginskey, 1993; Francia et al., 2001; Russell and Ginskey, 1995). We determined the direction of the maximum overshoot fields and found them to be parallel to M1. This feature suggests that one interprets the overshoot field in terms of a strengthening of the same current system related to the main variation (Russell and Ginskey, 1995). Some evidence for an overshoot structure with similar characteristics is observed in the night sector, while it almost disappears in the noon quadrant.

The overshoot peak is followed by large amplitude, almost regular fluctuations that appear in phase at each station and persist for a few cycles. This mode appears in the dusk sector, more clearly above $\sim 50^{\circ}$, and rapidly decreases below $\sim 40^{\circ}$ (Fig. 7). The spectral analysis (Fig. 8a) shows that these fluctuations consist of a dominant mode at an approximately constant frequency $(\sim 2.8 \mathrm{mHz})$. It is interesting to note that this peak has correspondence in the power spectrum of the $B_{z}$ component in the noon quadrant (Fig. 8b, Goes 8); it shows, indeed, a power enhancement which has approximately the same amplitude as in the low latitude ground spectra. Conversely, Goes 10 observations do not show any power enhancement in the early morning hours. Likely, these fluctuations might be related to the low frequency modes at discrete frequencies $(\sim 1.1,1.7,2.3,2.8,3.7 \mathrm{mHz})$ that have been observed from low to high-latitudes and interpreted in terms of ground signatures of magnetospheric cavity/waveguide modes (Kivelson and Southwood, 1985, 1986; Samson et al., 1992; Walker et al., 1992; Villante et al., 2001, 2003). On the other hand, in agreement with present results, recent investigations proposed much clearer evidence for power enhancements at selected frequencies in the afternoon sector and during higher pressure SW conditions; as a consequence, the onset of these fluctuations was related to the Earth's arrival of higher pressure corotating SW structures impinging on the postnoon magnetosphere (Villante et al., 2001, 2003).

As previously stated, the general aspects of the asymptotic variation have been currently investigated considering the geomagnetic response of the $H$ component approximately 10 min after the peak response (Russell et al., 1992, 1994a, b; Russell and Ginskey, 1995; Francia et al., 2001). In order to allow for a comparison with previous investigations, we conducted a similar analysis considering the difference between the 10-min average, 16:05-16:15 UT, and $\mathrm{H}_{E}$. As shown in the top panel of Fig. 9, this asymptotic response has the highest values in the dusk sector $\left(\sim 21-31 \mathrm{nT} /(\mathrm{nPa})^{1 / 2}\right.$, above $\left.\sim 40^{\circ}\right)$, where it shows a general, although irregular, tendency to increase with increasing latitude (Tsunomura, 1998); conversely, in the dawn and prenoon sectors, it is small for group $3 \mathrm{a}$ events and becomes negligible, or even negative, for group $3 \mathrm{~b}$ events. Although with few observations, our results seem to suggest approximately the same pattern at $\sim 30^{\circ}$, where the noon response $\left(\sim 11 \mathrm{nT} /(\mathrm{nPa})^{1 / 2}\right)$ is appreciably smaller than the midnight 

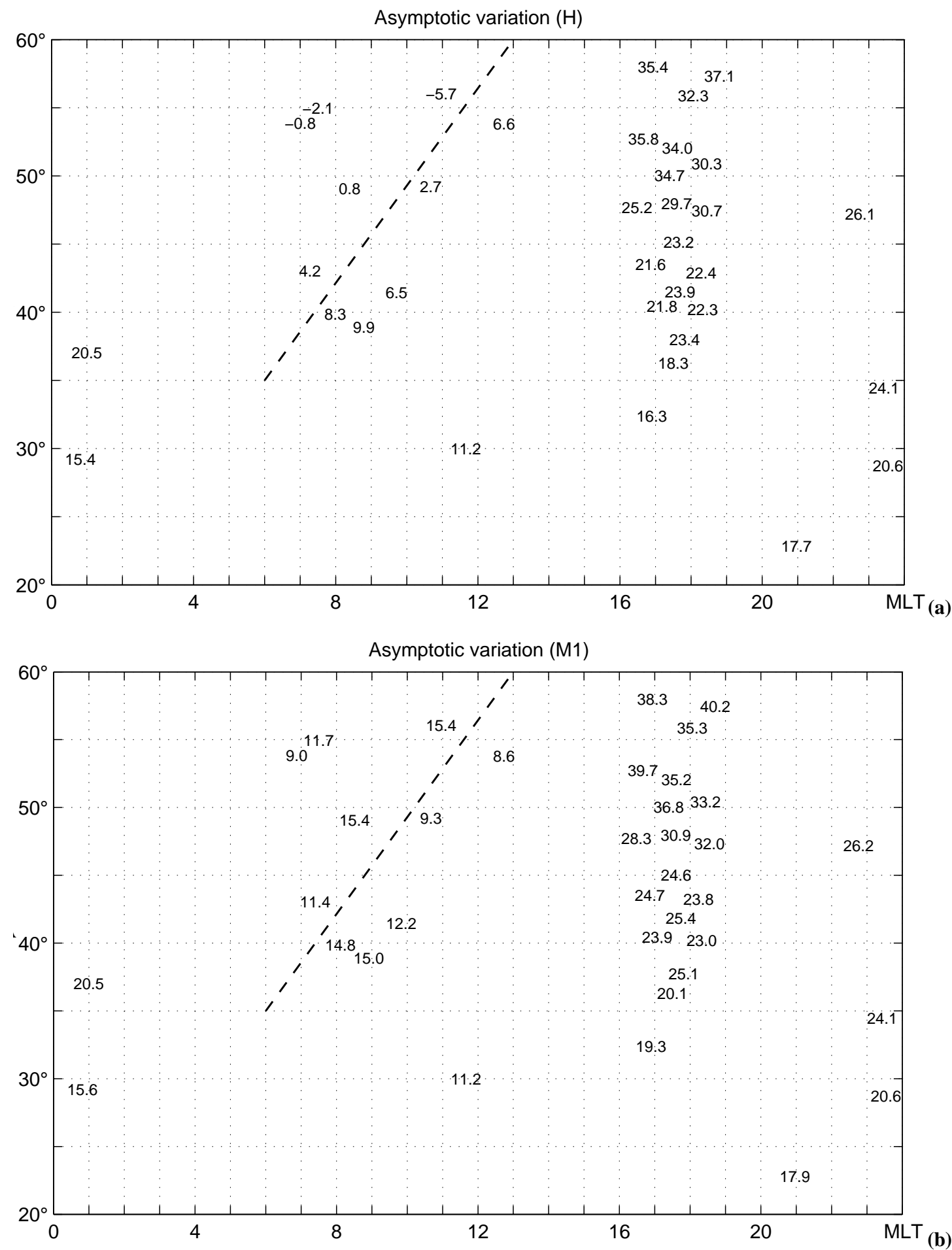

Fig. 9. The asymptotic variation (in $\mathrm{nT}$ ) at different ground stations. Top plot: $H$ component; bottom plot: the variation along the M1 axis.

response $\left(\sim 21 \mathrm{nT} /(\mathrm{nPa})^{1 / 2}\right)$. This aspect suggests that the lowest latitude observations cannot be interpreted in terms of the Chapman-Ferraro current alone. In this case, indeed, the noon response should be larger than the midnight response up to $\sim 45^{\circ}$ (Russell et al., 1994a).

On the other hand, the results of the present investigation suggest that an analysis of the $H$ component alone may provide erroneous conclusions on the amplitude and MLT dependence of the geomagnetic response. So, in the bottom panel of Fig. 9 we show the results obtained for the asymptotic variation along M1. Basically, they confirm a clear MLT variation with depressed prenoon values and an enhanced afternoon response; nevertheless, the global excursion is much smaller than for the $H$ component alone: 

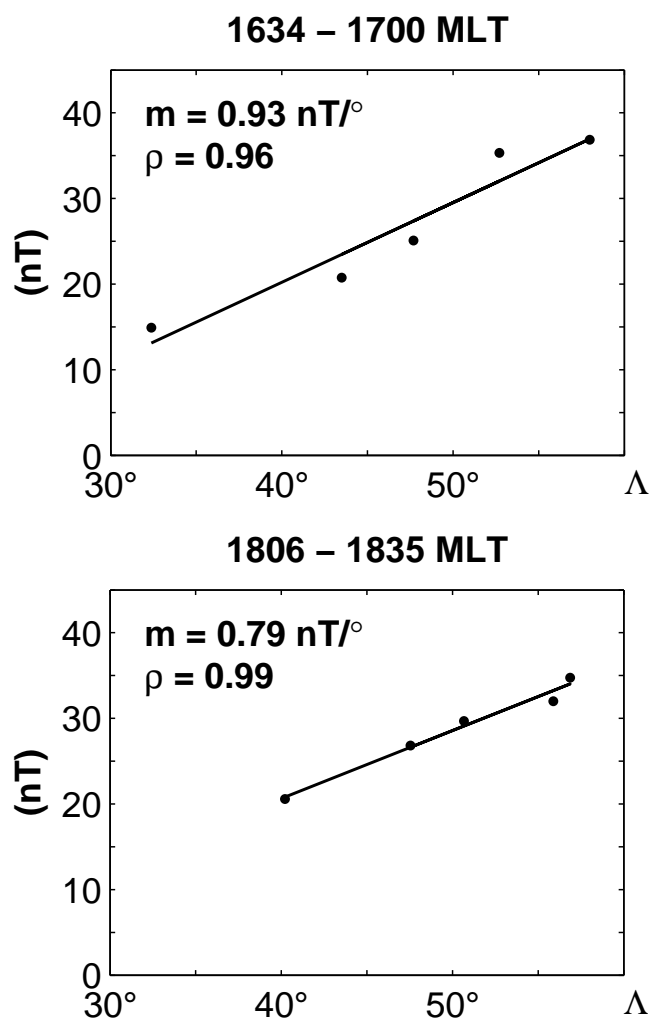

Fig. 10. The latitudinal variation of the asymptotic response along M1 in two narrow MLT strips in the dusk sector. $m$ is the angular coefficient.

indeed, above $\sim 40^{\circ}$, prenoon values range between 8.6 and $15.4 \mathrm{nT} /(\mathrm{nPa})^{1 / 2}$, while dusk values range between 23.0 and $40.2 \mathrm{nT} /(\mathrm{nPa})^{1 / 2}$. Note also that in the entire prenoon sector the geomagnetic response is smaller than in the noon quadrant at geostationary orbit $\left(\sim 18.7 \mathrm{nT} /(\mathrm{nPa})^{1 / 2}\right)$; conversely, it is explicitly larger in the dusk and night sectors.

The aspects of the latitudinal variation, in the dusk sector, have been better evaluated by focusing attention on two narrow MLT strips (Fig. 10): as a matter of fact we found a strong correlation between the field variation and geomagnetic latitude, with an average latitudinal gradient that seems to decrease at later MLTs (namely, $\sim 0.93 \mathrm{nT} /{ }^{\circ}$ at $\sim 16: 45 \mathrm{MLT} ; \sim 0.79 \mathrm{nT} /{ }^{\circ}$ at $\left.\sim 18: 15 \mathrm{MLT}\right)$. It is interesting to remark that approximately the same latitudinal gradient for the $H$ component can be inferred by the experimental results obtained by Petrinec et al. (1996) between $30^{\circ}$ and $60^{\circ}$, around dusk.

\section{Discussion and conclusions}

In the present paper we examined several aspects of the magnetospheric and geomagnetic response to the variable SW conditions observed during a 50-min interval. We focused particular attention on the correspondence between different $\mathrm{SW} /$ magnetospheric structures and geomagnetic variations. We carefully examined the aspects of the MLT and latitu- dinal dependence of the geomagnetic response at low and middle latitudes. We also proposed a new approach to the combined $H$ and $D$ data analysis. This approach allows for a clearer identification of the global geomagnetic response and a better understanding of the results obtained at different sites. On the other hand, this event occurred in the recovery phase after the day the SW almost disappeared: in this sense our results can be useful to examine the aspects of the geomagnetic response soon after a huge expansion of the Earth's magnetosphere.

The different aspects of the geomagnetic response (Matsushita, 1962; Nishida and Jacobs, 1962) reveal in this case a clear organization in four groups of events. The differences between groups include, in our opinion, several aspects of the more generic "morning/afternoon asymmetry" proposed by previous analysis. Indeed, a visual inspection of the experimental observations reported in the scientific literature suggests that, as for the present case, the transition between structures with different characteristics of the $H$ component typically occurs at later MLTs for higher latitudes (namely, in the early morning hours at $\sim 30^{\circ}-35^{\circ}$, around noon at $\sim 55^{\circ}-$ $60^{\circ}$; Le et al., 1993; Russell and Ginskey, 1995; Tsunomura, 1998). Our results also reveal that the different responses at different sites basically reflect a close correspondence between the magnetospheric and the ground signals which occur along an axis that progressively rotates from $\mathrm{N} / \mathrm{S}$ (night events) to an E/W orientation (dawn events). We concluded that a simple two vortex system at these latitudes interprets well all the aspects of the MLT and latitudinal dependence, without requesting additional contributions (Russell and Ginskey, 1995): indeed, in agreement with the inferred currents, a similar MLT pattern progressively emerges from lower to higher latitudes, with an amplitude modulation much smaller than previously estimated; in addition, a sharp latitudinal dependence is detected in the dusk sector where current lines are perpendicular to geomagnetic meridians.

This event occurred after a prolonged interval of extremely rarefied SW conditions which provided a huge extension of the Earth's magnetosphere (up to $\sim 60 R_{e}$ at $\sim$ 19:00 UT, 11 May, Le et al., 2000). The density started to recover after $\sim$ 20:00 UT, reaching $\sim 20 \mathrm{~cm}^{-3}$ on 12 May at $\sim 18: 00$ UT. Several aspects of the magnetospheric response have been examined in the scientific literature for such peculiar SW conditions. Namely, at geostationary orbit the magnetospheric field became closely dipolar for $\sim 16 \mathrm{~h}$ (11 May, 12:00 UT-12 May, 04:00 UT, Farrugia et al., 2000); the ground magnetic field disturbances due to the ring current and the magnetopause currents decreased on 11 May to values substantially smaller than quiet times values $\left(K_{p}=0^{+}\right.$, $D_{s t}$ between -4 and $10 \mathrm{nT}$, Jordanova et al., 2001); the pattern of the field-aligned currents, at the maximum magnetospheric expansion, was significantly rotated toward dusk: indeed, while the typical R1/R2 current system was observed near dawn, the R0/R1 current system, which is typical of the noon quadrant, was observed near dusk (Othani et al., 2000). Our results allow one to conclude that, on 12 May , 15:00 UT, the magnetosphere had already restored 
its typical conditions; indeed that, as previously discussed, most aspects of the geomagnetic response appear consistent with a familiar current system. In this sense our conclusions are consistent with Wind observations that suggest a magnetosonic Mach number of $\sim 2$ in the morning hours and increasing to $\sim 8-10$ in the afternoon hours (Farrugia et al., 2000). Indirectly, interesting results in this sense also come from an analysis of the long period fluctuations detected during different time intervals on 12 May. Indeed, the high-latitude observations from IMAGE magnetometer array revealed, between $\sim$ 03:00-09:00 UT, prominent spectral peaks at $\sim 0.8 \mathrm{mHz}$ and $\sim 2.2 \mathrm{mHz}$ (Mathie and Mann, 2000). The latitudinally localized amplitude of these signals and their morphological characteristics were interpreted in terms of field line resonances coupling with discrete cavity/waveguide modes of the entire magnetosphere: nevertheless, the observed frequencies were significantly shifted to lower values than cavity modes frequencies and suggested a still extended magnetosphere supporting unusual cavity modes. Conversely, a few hours later, our analysis of the wave mode following the SI confirms restored magnetospheric dimensions: indeed, the experimental observations were consistent with a global magnetospheric mode matching one of the usual cavity mode frequencies $(\sim 2.8 \mathrm{mHz})$.

The orientation and latitudinal dependence of the overshoot field suggests a transient modification of the highlatitude vortex system in the dusk sector. Our results can be considered consistent with theoretical models (Osada, 1992; Araki, 1994) which interpret such overshoot fields (increasing with latitude) in terms of the arrival of compressional waves travelling along magnetospheric field lines. On the other hand, overshoot amplitudes are much greater than in other cases. This aspect might be considered consistent with the significant reduction of the ring current in the period of interest (on 12 May, the $D_{s t}$ index progressively increased from 2 to $37 \mathrm{nT}$ between 10:00-18:00 UT, and attained values of $31 \mathrm{nT}$ at 16:00 UT): Russell and Ginskey (1993), indeed, speculated that a stronger ring current would correspond to a higher damping of propagating compressional waves, with a consequent reduction of overshoot amplitudes.

Acknowledgements. The key parameters of Wind MFI and SWE, Goes 8 and Goes 10 magnetometers were provided by the NASA Goddard Space Flight Center web site. The ground data are from the CD-ROM of INTERMAGNET "Magnetic Observatory Definitive Data", 1999. Authors are grateful to principal investigators of space and ground experiments. This research activity is supported by MIUR and ASI.

Topical Editor T. Pulkkinen thanks H. Lühr and another referee for their help in evaluating this paper.

\section{References}

Araki, T. and Nagano, H.: Geomagnetic response to sudden expansions of the magnetosphere, J. Geophys. Res., 93, 3983-3988, 1988.

Araki, T.: A physical model of the geomagnetic sudden commencement, in Solar Wind Sources of Magnetospheric UltraLow-Frequency Waves, Geophys. Monogr. Ser., vol. 81, edited by Engebretson, M. J.,Takahashi, K., and Scoler, M., pp. 183200, AGU, Washington, D.C., 1994.

Araki, T., Fujitani, S., Emoto, M., Yumoto, K., Shiokawa, K., Ichinose, T., Lühr, H., Orr, D., Milling, D. K., Singer, H., Rostoker, G., Tsunomura, S., Yamada, Y., and Liu,,C. F.: Anomalous sudden commencements on March 24, 1991, J. Geophys. Res., 102, 14 075-14 086, 1997.

Fairfield, D. H., Cairns, I. H., Desch, M. D., Szabo, A., Lazarus, A. J., and Aellig, M. R.: The location of low Mach number bow shocks at Earth, J. Geophys. Res., 106, 25 361-25 376, 2001.

Farrugia, C. J., Freeman, M. P., Cowley, S. W. H., Southwood, D. J., Lockwood, M., and Etemadi, A.: Pressure-driven magnetopause motions and attendant response on the ground, Planet. Space Sci., 37, 589-607, 1989.

Farrugia, C. J., Singer, H. J., Evans, D., Berdichevsky, D., Scudder, J. D., Ogilvie, K. W., Fitzenreiter, R. J., and Russell, C. T.: Response of the equatorial and polar magnetosphere to the very tenuous solar wind on 11 May 1999, Geophys. Res. Lett., 27, 3773-3776, 2000

Francia, P., Lepidi, S., Villante, U., Di Giuseppe, P., and Lazarus, A. J.: Geomagnetic response at low latitude to continuous solar wind pressure variations during northward interplanetary magnetic field, J. Geophys. Res., 104, 19923-19930, 1999.

Francia, P., Lepidi, S., Di Giuseppe, P., and Villante, U.: Geomagnetic sudden impulses at low latitude during northward interplanetary magnetic field conditions, J. Geophys. Res., 106, 21 231-21 236, 2001.

Francia, P., and Lepidi, S.: Latitudinal dependence of the geomagnetic response to solar wind pressure variations during the earth's passage of the 15-16 July 2000 coronal ejecta, Solar cycle and space conference proceedings, Vico Equense (Italy), 24-29 September 2001, ESA SP-477, pp. 431-434, 2002.

Jordanova, V. K., Farrugia, C. J., Fennell, J. F., and Scudder, J. D. Ground disturbances of the ring, magnetopause, and tail currents on the day the solar wind almost disappeared, J. Geophys. Res., 106, 25 529-25 540, 2001.

Kivelson, M. and Southwood, D. J.: Resonant ULF waves: A new interpretation, Geophys. Res. Lett., 12, 49-52, 1985.

Kivelson, M. and Southwood, D. J.: Coupling of global magnetospheric MHD eigenmodes to field line resonances, J. Geophys. Res., 91, 4345-4351, 1986.

Kokubun, S.: Characteristics of storm sudden commencement at geostationary orbit, J. Geophys. Res., 88, 10 025-10 033, 1983.

Kuwashima, M., and Fukunishi, H.: Local time asymmetries of the SSC-associated hydromagnetic variations at the geosynchronous altitude, Planet. Space Sci., 33, 711-720, 1985.

Le, G., Russell, C. T., Petrinec, S. M., and Ginskey, M.: Effect of sudden solar wind dynamic pressure changes at subauroral latitudes: Change in magnetic field, J. Geophys. Res., 98, 39833990, 1993.

Le, G., Russell, C. T., and Petrinec, S. M.: The magnetosphere on 11 May 1999: the day the solar wind almost disappeared: I current systems, Geophys. Res. Lett., 27, 1827-1830, 2000. 
Mathie, R. A. and Mann, I. R.: Observations of an anomalously low frequency Alfven continuum in an abnormally expanded magnetosphere, Geophys. Res. Lett., 27, 4017-4020, 2000.

Matsushita, S.: On geomagnetic sudden commencements, sudden impulses, and storm durations, J. Geophys. Res., 67, 3753-3777, 1962.

Nishida, A., and Jacobs, J. A.: World-wide changes in the geomagnetic field, J. Geophys. Res., 67, 525-540, 1962.

Nishida, A.: Geomagnetic Dp2 fluctuations and associated magnetospheric phenomena, J. Geophys. Res., 73, 1795-1803, 1968.

Nishida, A.: Geomagnetic diagnoses of the magnetosphere, Springer-Verlag, New York, 1978.

Osada, S.: Numerical calculation of geomagnetic sudden commencement, Master Thesis, Faculty of Science, Kyoto Univ., March 1992.

Othani, S., Newell, P. T., and Takahashi, K.: Dawn-dusk profile of field-aligned currents on 11 May 1999: A familiar pattern driven by an unusual case, Geophys. Res. Lett., 27, 3777-3780, 2000.

Papitashvili, V. O., Clauer, C. R., Christiansen, F., Pilipenko, V. A., Popov, V. A., Rasmussen, O., Suchdeo, V. P., and Watermann, J. F.: Geomagnetic disturbances at high-latitudes during very low solar wind density event, Geophys. Res. Lett., 23, 3785-3788, 2000.

Petrinec, S. M., Yumoto, K., Lühr, H., Orr, D., Milling, D., Hayashi, K., Kokubun, S., and Araki, T.: The CME event of 21 February 1994: response of the magnetic field at the Earth's surface, J. Geomag. Geoelectr., 48, 1341-1379, 1996.

Rostoker, G.: Ground magnetic signatures of ULF and substorm activity during an interval of abnormally weak solar wind on May 11, 1999, Geophys. Res. Lett., 27, 3789-3792, 2000.

Russell, C. T., Ginskey, M., Petrinec, S., and Le, G.: The effect of solar wind dynamic pressure changes on low and mid-latitude magnetic records, Geophys. Res. Lett., 19, 1227-1230, 1992.

Russell, C. T., and Ginskey, M.: Sudden impulses at low latitudes: transient response, Geophys. Res. Lett., 20, 1015-1018, 1993.

Russell, C. T., Ginskey, M., and Petrinec, S. M.: Sudden impulses at low-latitude stations: Steady state response for northward interplanetary magnetic fields, J. Geophys. Res., 99, 253-261, 1994a.

Russell, C. T., Ginskey, M., and Petrinec, S. M.: Sudden impulses at low-latitude stations: steady state response for southward interplanetary magnetic fields, J. Geophys. Res., 99, 13 403-13 408, 1994b.
Russell, C. T. and Ginskey, M.: Sudden impulses at subauroral latitudes: response for northward interplanetary magnetic field, J. Geophys. Res., 100, 23 695-23 702, 1995.

Samson, J. C., Harrold, B. G., Ruohoniemi, J. M., Greenwald, R. A., and M. Walker, A. D.: Field line resonances associated with MHD waveguides in the magnetosphere, Geophys. Res. Lett., 19, 441-444, 1992.

Sastri, J. H., Takeuchi, T., Araki, T., Yumoto, K., Tsunomura, S., Tachihara, H., Lühr, H., and Watermann, J.: Preliminary impulse of the geomagnetic storm sudden commencement of November 18, 1993, J. Geophys. Res., 106, 3905-3918, 2001.

Siscoe, G. L.,Formisano, V., and Lazarus, A. J.: Relation between geomagnetic sudden impulses and solar wind pressure changes: An experimental investigation, J. Geophys. Res., 73, 4869-4874, 1968.

Su, S. and Konradi, A.: Magnetic field depression at the Earth's surface calculated from the relationship between the size of the magnetosphere and the $D_{s t}$ values, J. Geophys. Res., 80, 195199, 1975.

Terasawa, T., Kasaba, Y., Tsubouchi, K., Mukai, T., Saito, Y., Frank, L. A., Paterson, W. R., Ackerson, K., Matsumoto, H., Kojima, H., Matsui, H., Larson, D., Lin, R., Phan, T., Steinberg, J., McComas, D., Skoug, R., Fujimoto, M., Hoshino, M., and Nishida, A.: GEOTAIL observations of anomalously low density plasma in the magnetosheath, Geophys. Res. Lett., 23, 3781-3784, 2000.

Tsunomura, S.: Characteristics of geomagnetic sudden commencement observed in middle and low latitudes, Earth Planets and Space, 50, 755-772, 1998.

Villante, U., Francia, P., and Lepidi, S.: Pc5 geomagnetic field fluctuations at discrete frequencies at a low latitude station, Ann. Geophysicae, 19, 321-325, 2001.

Villante, U., Francia, P., Vellante, M., and Di Giuseppe, P.: Some aspects of the low latitude geomagnetic response under different solar wind conditions, Space Science Rev., 107, 207-217, 2003.

Walker, A. D. M., Ruohoniemi, J. M., Baker, K. B., Greenwald, R. A., and Samson, J. C.: Spatial and temporal behavior of ULF pulsations observed by the Goose Bay HF radar, J. Geophys. Res., 97, 12 187-12 202, 1992. 